\title{
The Effect of Health Insurance on Emergency Department Visits: Evidence from an Age-Based Eligibility Threshold
}

\author{
Michael Anderson \\ UC Berkeley \\ mlanderson@berkeley.edu
}

\author{
Carlos Dobkin \\ UC Santa Cruz \& NBER \\ cdobkin@ucsc.edu
}

\author{
Tal Gross \\ Columbia University \\ tg2370@columbia.edu
}

April 13, 2012

\begin{abstract}
This paper studies how health insurance affects the rate at which individuals visit hospitals and emergency departments (EDs). We identify the causal effect of losing health insurance using a regression discontinuity design. We compare individuals just before and just after their $23^{\text {rd }}$ birthdays, which private insurers have used as a cutoff after which students are no longer eligible for their parents' health insurance. Roughly 1.5 percent of young adults lose their health insurance on their $23^{\text {rd }}$ birthday, and we find that this transition leads to a 1.6-percent decrease in ED visits and a 0.8percent decrease in visits to the hospital. We discuss why these estimates are significantly larger than those observed among teenage populations.
\end{abstract}

JEL Codes: I11, I18, G22

Keywords: Health insurance, health care utilization

\footnotetext{
* We thank Jan Morgan at the California Office of Statewide Health Planning and Development for assistance with the California hospital discharge records. All mistakes are our own.
} 


\section{INTRODUCTION}

Two central goals of health care reform in the United States are to expand health insurance coverage and to restrain growth in medical expenditures. Legislation passed in 2010 relies on several mechanisms to expand insurance coverage, but it is unclear how this legislation will affect expenditures. A large body of observational studies suggests that an expansion of insurance coverage could increase health care utilization (Institute of Medicine 2002). However, since health insurance is not randomly assigned, drawing causal inferences from these studies is challenging.

In this paper, we exploit quasi-random variation in insurance status that results from rules on which private insurers rely. Many private health insurance contracts cover dependents that are full-time students through age 23 (Marquis and Long 1995; White 2002). As a result, many young adults become uninsured at the end of the month in which their $23^{\text {rd }}$ birthday occurs. We use the abrupt decrease in insurance coverage induced by these rules to estimate a regression discontinuity (RD) design. Under this design we compare the health care consumption of individuals who are slightly younger than age 23 to the health care consumption of individuals who are slightly older than age 23. These comparisons should estimate causal effects as long as other factors affecting health care consumption do not change abruptly at age 23 .

Our study is not alone in estimating the causal effect of health insurance on utilization. Several studies focus on Medicare or Medicaid (Currie and Gruber, 1996; Finkelstein 2007; Card et al. 2008, 2009; Currie et al. 2008). More recently, Finkelstein et al. (2011) examine a lottery in Oregon in which uninsured, low-income adults were randomly selected for Medicaid coverage, and Kolstad and Kowalski (2010) and Miller (2011) study how universal health insurance in Massachusetts has affected utilization. In a related paper, Anderson et al. (2012), we exploit a similar transition at age 19 to estimate the impact of health insurance on utilization for young adults who stop attending school prior to turning 19.

This paper builds upon previous studies in several key respects. First, while the seminal work on Medicare and Medicaid illustrates the impact of these programs, it provides limited 
insight regarding the likely effects of coverage expansions on currently uninsured young adults. In particular, the previous literature focuses on the near elderly or the very young, while most of those currently uninsured are non-elderly adults (Kriss et al. 2008). Second, in contrast to previous studies, this paper estimates effects for mostly college-educated young adults. It also focuses on the effects of private health insurance when estimating the impacts on emergency department (ED) visits. Finally, this paper's results are directly applicable to a recent policy change that mandates coverage of dependents through age 26 . The legislation enacting this policy is the subject of continued public and legal debate.

We implement an RD design using ED administrative records from five states and hospital admission records from six states. We find that losing private coverage generates large reductions in $\mathrm{ED}$ visits, contradicting the conventional wisdom that low rates of health insurance coverage contribute substantially to ED crowding (Newton et al. 2008). We also find that losing private coverage induces significant transitions in coverage among hospital inpatients and causes a decline in scheduled inpatient visits. However, the exact mechanism by which inpatient visits drop is unclear, as many inpatients that lose private coverage transition onto Medicaid. These results suggest that recent policies extending coverage of all dependents to age 26 could increase the amount of ED and inpatient care consumed by young adults.

\section{The EfFects of Health Insurance on EMERgenCy Department Visits}

Young adults frequently seek care in hospital emergency departments. Over one quarter of 22-year-old respondents to the National Health Interview Survey (NHIS) report receiving treatment in an ED in the prior 12 months. ${ }^{1}$ Two factors make ED utilization a topic of policy interest. First, ED crowding is associated with long waits and hospital diversions, both of which are believed to negatively affect patient care (Fatovich 2002; Trzeciak and Rivers 2003; Kellermann 2006). How health insurance affects ED utilization will determine whether insurance coverage expansions alleviate or exacerbate ED crowding. Second, providing care in the ED is expensive. Bamezai et al. (2005) estimate that the marginal cost of a non-trauma $\mathrm{ED}$ visit is $\$ 300$, a number that is several times higher than the average price of a doctor's

\footnotetext{
1 Authors' calculations from the NHIS.
} 
visit (let alone the marginal cost). ${ }^{2}$ The effect of insurance on ED usage is thus an important component of the cost of insurance coverage expansions.

Figure 1 presents the age profile of insurance status for 22- to 24-year old ED patients. The underlying data represent a near census of ED visits in Arizona, California, Iowa, New Jersey, and Wisconsin from 2004 to 2009 (coverage dates vary by state - see Table 1 note for details). Panels A through $\mathrm{D}$ plot the share of patients with each type of insurance coverage by age in months - each point represents the average coverage level in a one-month age cell. The plots demonstrate a discontinuous reduction in private insurance coverage immediately after young adults turn 23 (Panel B) and a corresponding increase in the proportion uninsured (Panel A). The share of patients who are privately insured decreases by 0.9 percentage points, and the proportion uninsured rises by 0.8 percentage points. There are no visible changes in the proportions covered by Medicaid or other insurance. This suggests that the transition at age 23 is driven by a change in private insurance coverage and is not confounded by simultaneous changes in public insurance coverage.

These estimates, however, understate the true change in coverage. This bias stems from sample selection: we only observe insurance status for individuals that visit the ED. Below we demonstrate that losing insurance reduces the likelihood of an ED visit and thus affects the probability of appearing in the sample. The population of individuals visiting the ED just after turning 23 is therefore not directly comparable to the population of individuals visiting the ED just before turning 23. This selection mechanism attenuates the estimates in Panels $\mathrm{A}$ and $\mathrm{B}$ of Figure 1, because many of those who have lost insurance coverage are likely to drop out of the sample. The actual share of the population that has lost insurance coverage at age 23 is thus even larger than appears in the ED data.

In Anderson et al. (2012) we derive a procedure that corrects for this bias. The intuition underlying this adjustment is the following. The instrumental variables (IV) exclusion restriction implies that the only reason ED visits change at age 23 is through a loss of insurance coverage. We can thus assume that the observed reduction in ED visits at age 23, which is a population-level estimate unaffected by the sampling issue, occurs exclusively

\footnotetext{
2 The average total payment for a doctor visit recorded in the Medical Expenditure Panel Survey is $\$ 120$.
} 
among individuals losing insurance coverage. We can then "add back in" these individuals when calculating the true change in insurance coverage at age 23 , coding all of them as uninsured. ${ }^{3}$ Doing so increases the estimated change in the proportion uninsured.

The first row of Table 1 presents estimates of the change in insurance coverage at age 23 in the ED after the bias adjustment. As in Figure 1, we estimate the regressions using the proportions from 25 one-month age cells. Private coverage falls by 1.5 percentage points at age $23(t=9.3)$, and the proportion uninsured rises by 1.8 percentage points $(t=8.2)$. ${ }^{4}$ There are no significant changes in Medicaid or other insurance coverage.

The final panel of Figure 1 presents the age profile of ED visit rates. A visit rate of 3,300 per 10,000 person years implies that the average person visits the ED 0.33 times per year. ${ }^{5}$ The figure reveals a discontinuous reduction in the ED visit rate at age 23 . There is no notable increase in $\mathrm{ED}$ visits in the month before the $23^{\text {rd }}$ birthday, suggesting that individuals do not "stock up" on ED care right before their insurance lapses. The first row of Table 2 presents the analogous regression estimates. The regressions suggest a 1.6 percent decrease in ED visits at age $23(t=5.3)$. Table 2 also reports this estimate by hospital type. We estimate that ED visits fall by 1.5 percent at non-profit hospitals $(t=4.3)$ and 3.2 percent at for-profit hospitals $(t=3.6)$. However, visits at public hospitals fall by only 0.9 percent $(t=$ 1.1). Many public hospitals are designated "core safety net providers" that render services to low-income individuals at reduced cost. The weaker effect at public hospitals is thus consistent with a weaker effect of insurance coverage on prices that patients face at these hospitals.

These reduced-form estimates measure the percent change in ED visits occurring at age 23. We generate IV estimates by dividing the percent change in visits from Table 2 (the reduced

${ }^{3}$ See Anderson et al. (2012) for a detailed description of this procedure and the technical proof of its validity.

4 We estimate a 1.6 percent reduction in admissions at age 23 (described below), and this effect shifts the estimated change in the proportion privately insured from 0.9 percentage points to 1.5 percentage points. It also shifts the estimated change in the proportion uninsured from 0.8 percentage points to 1.8 percentage points. The adjustment is greater for the proportion uninsured than for the proportion privately insured because the number of "missing" individuals is a larger share of the number of privately insured than the number of uninsured.

5 The numerator for the rates comes from a near census of ED visits from Arizona, California, Iowa, New Jersey, and Wisconsin. The denominator comes from annual estimates of the resident populations of states by gender and age, published by the US Census. 
form) by the estimates of the percentage-point change in insurance coverage rates from Table 1 (the first stage). Table 3 presents such estimates. Overall, the implied IV estimate is $-0.87(t=-4.4)$. This implies that a 10 -percentage-point increase in the insurance coverage rate among young adults would increase ED visits by nearly 9 percent. The estimates vary by hospital ownership, with the strongest effects at for-profit hospitals $(-1.76)$ and statistically insignificant effects at public hospitals $(-0.50)$.

\section{The EFFECTS OF THE INSURANCE Transition ON INPATIENT ADMISSIONS}

Inpatient visits to the hospital are less common than ED visits. Approximately 6 percent of young adults have had an inpatient admission in the past year. ${ }^{6}$ Nevertheless, such visits are expensive; approximately 34 percent of total health care spending is driven by inpatient admissions. ${ }^{7}$ As such, the effect of insurance coverage on inpatient visits is an object of critical interest.

The first four panels of Figure 2 present the age profile of insurance coverage among nonpregnant 22- to 24-year-olds admitted to the hospital. The underlying data represent a near census of inpatient visits in Arizona, California, Iowa, New York, Texas and Wisconsin from 1990 to 2009 (coverage dates vary by state - see Table 1 note for details). Overall, the share of patients who are uninsured is far lower than the share observed in the ED (32 percent versus 21 percent). Panel B reveals a sharp decline in private coverage at age 23 . This decline is matched by a modest increase in the proportion uninsured (Panel A) and sharper increase in the proportion covered by Medicaid (Panel C). The increase in Medicaid coverage likely occurs because hospitals have strong incentives to enroll newly uninsured patients in Medicaid if there is any chance that they will qualify.

The estimates of the change in insurance coverage at age 23 are biased by a change in composition similar to the one that affects the ED estimates. The second row of Table 1 presents estimates of the discontinuous change in insurance coverage that occurs at age 23 , corrected for bias in the manner described in the prior section. The estimates reveal a 1.7

6 Authors' calculations from the NHIS.

${ }^{7}$ Authors' calculations from the Medical Expenditure Panel Survey. 
percentage point decrease in private insurance coverage $(t=7.4)$ that is similar in magnitude to that observed in the ED. Unlike the ED estimates, however, we observe a 0.6 percentage point increase in the share of patients covered by Medicaid $(t=2.7){ }^{8}$ The proportion uninsured increases 1.1 percentage points $(t=4.0)$.

The simultaneous changes in the proportion privately insured and the proportion covered by Medicaid make it difficult to isolate the relative effect of each insurance type on inpatient visits. We thus focus on the reduced form effect of the policy change at age 23 on inpatient visits. Panels $\mathrm{E}$ through $\mathrm{G}$ of Figure 2 present the reduced form graphically. Panel E suggests a modest but statistically significant drop in inpatient visits at age 23 . We divide inpatient visits into two categories: those in which the patient was transferred from the ED and those in which the patient was admitted to the hospital directly. The latter are generally scheduled procedures and are more likely to be discretionary. We plot both types of visits in the final panels of Figure 2. The plots suggest that any decrease in inpatient visits is driven by scheduled visits, rather than by inpatient visits that originated in the ED. This finding is consistent with our previous work (Anderson et al. 2012), in which the largest effects occurred for inpatient visits that did not originate in the ED. Despite the discretionary nature of these visits, there is no evidence that individuals stockpile care right before coverage lapses, as inpatient visits are not above trend in the month prior to turning 23.

The last three rows of Table 2 present the regression estimates that underlie these figures. We observe a statistically significant 0.8 percent decrease in all inpatient visits $(t=2.3)$ and a 2.3 percent decrease in inpatient visits that were not through the $\mathrm{ED}(t=3.6)$. In contrast, we observe no statistically significant change in inpatient visits that originated in the ED. The decrease in visits is largest in private, for-profit hospitals (3.5 percent for all inpatient visits and 7 percent for inpatient visits not through the ED). The decrease in total inpatient visits is statistically insignificant in other hospitals, though inpatient visits not through the ED significantly decrease in private, non-profit hospitals.

\footnotetext{
8 The share covered by Medicaid may increase by more than 0.6 percentage points if some individuals who avoid visiting the hospital because they lost private insurance coverage sign up for Medicaid instead of remaining uninsured. However, it seems unlikely that an individual would make the effort to enroll in Medicaid and then promptly choose not to use it.
} 


\section{Discussion}

We estimate a large, statistically significant decrease in private insurance coverage at age 23 and accompanying decreases in ED visit rates and hospital inpatient admission rates. These estimates represent causal effects of a reduction in private insurance coverage under the assumption that no other policy changes occur directly after individuals turn 23 . This seems particularly likely since most age-related events (e.g., college graduation) occur at a particular time of year (e.g., June), while $23^{\text {rd }}$ birthdays are distributed throughout the year.

Though they are likely consistent, our estimates are local along two dimensions. First, they apply specifically to people around 23 years old. Second, the IV estimates (Table 3) apply only to "compliers" - individuals who lose coverage due to the age 23 provision. The compliers are students who are covered under their parents' health plans and do not have an alternate source of coverage. In spite of these limitations, however, our estimates are policy relevant for two reasons.

First, recent legislation mandates that dependents - including those that are married, do not live with their parents, or are not dependents for tax purposes - may remain on their parents' insurance plans through age 26. Our results imply that this provision should increase insurance coverage and ED and inpatient visit rates among individuals in their midtwenties. This is true even in states that force insurers to cover dependents older than 23 , as many companies self-insure and are therefore exempt from state insurance regulations (Levine et al. 2011). ${ }^{9}$

Second, our results represent the first quasi-experimental estimates of the effects of private insurance coverage on ED use. Previous studies, including those that focus on Medicare, Medicaid, and the Massachusetts health reform, have estimated the effects of public

\footnotetext{
${ }^{9}$ State policies mandating coverage through specific ages are implemented by state insurance regulators. These regulators do not have jurisdiction over firms that self-insure, as there is no formal insurance product being bought or sold. Recent Federal legislation, the Patient Protection and Affordable Care Act of 2010 (PPACA), mandates that dependents may remain on their parents' insurance plan until their 26th birthday. This provision applies to all firms, including those that self-insure.
} 
insurance coverage. Our estimates complement those studies and demonstrate that private insurance coverage can sharply increase ED utilization.

It is interesting to compare this paper's ED utilization estimates to those generated by the reduction in insurance coverage at age 19 (Anderson et al. 2012). At age 19, private insurance coverage falls by 6.3 percentage points, Medicaid coverage falls by 1.7 percentage points, and ED visits fall by 3.3 percent. Those results imply that transitioning to an uninsured state from a mixture of private and public coverage reduces ED visits by 40.4 percent ( 95 percent confidence interval: 24.2-56.6). In comparison, our results at age 23 imply that transitioning to an uninsured state from private coverage reduces ED visits by 86.7 percent (95 percent confidence interval: 45.6-127.8). We can reject the hypothesis that the two estimates are equal at the 5-percent level, implying that the response to losing insurance coverage is stronger at 23 than at 19 . The different responses are likely due in part to differences in the composition of compliers at each age. Individuals who lose coverage at age 19 are of lower socioeconomic status than those who lose coverage at age 23 - they are not in school and are not working at jobs with health insurance benefits. It is possible that those losing coverage at age 19 are more likely to qualify for means-tested programs or are less concerned about the financial consequences of receiving care not covered by health insurance.

Our findings imply that extending the "age out" threshold to age 26 should increase use of ED care and scheduled inpatient care among young adults. To understand the potential magnitude of the policy's effect, we perform a simple back-of-the-envelope calculation. First, using NHIS data we calculate the difference between the proportion uninsured at ages 19-26 (29.4 percent) and the proportion uninsured at age 18 (16.8 percent). Since almost all employer-provided policies cover dependents through age 18, we interpret this difference (12.6 percentage points) as the share of young adults who could gain coverage through their parents' policies under the new law. Next, we make two assumptions about insurance takeup among newly eligible young adults. Our "low" estimate assumes 30 percent of eligible young adults take up their parents' insurance, while our "high" estimate assumes 70 percent of eligible young adults take up their parents' insurance. Finally, we apply an estimated elasticity of ED utilization with respect to insurance. Our "low" estimate comes from the age-19 discontinuity (0.404), and our "high" estimate comes from age-23 discontinuity 
(0.867). ${ }^{10}$ Our calculations suggest that the age-26 policy would increase annual ED visits by at least 143,000 visits and as much as 718,000 visits. These figures represent a 1.4 to 6.8 percent increase in the total number of ED visits by 19-26-year olds.

\section{REFERENCES}

Anderson, Michael, Carlos Dobkin, and Tal Gross. 2012. "The Effect of Health Insurance Coverage on the Use of Medical Services." American Economic Journal: Economic Policy 3(4): 1-27.

Bamezai, Anil, Glenn Melnick, and Amar Nawathe. 2005. “The cost of an emergency department visit and its relationship to emergency department volume." Annals of Emergency Medicine 45(5): 483-490.

Card, David, Carlos Dobkin, and Nicole Maestas. 2009. "Does Medicare Save Lives?" Quarterly Journal of Economics 124(2): 597-636.

— 2008. "The Impact of Nearly Universal Insurance Coverage on Health Care Utilization: Evidence from Medicare." American Economic Review.

Currie, Janet, Sandra Decker, and Wanchuan Lin. 2008. "Has Public Health Insurance for Older Children Reduced Disparities in Access to Care and Health Outcomes?" Journal of Health Economics 27(6): 1567-1581.

Currie, Janet, and Jonathan Gruber. 1996. "Health Insurance Eligibility, Utilization of Medical Care, and Child Health.” The Quarterly Journal of Economics 111(2): 431 -466.

Fatovich, Daniel M. 2002. "Recent developments: Emergency medicine.” BMJ : British Medical Journal 324(7343): 958-962.

Finkelstein, Amy. 2007. "The Aggregate Effects of Health Insurance: Evidence from the Introduction of Medicare." The Quarterly Journal of Economics 122(1-37).

Finkelstein, Amy et al. 2011. "The Oregon Health Insurance Experiment: Evidence from the First Year.” National Bureau of Economic Research Working Paper Series No. 17190.

Institute of Medicine. 2002. Care without coverage: too little, too late. National Academies Press.

Kellermann, Arthur L. 2006. "Crisis in the emergency department." The New England Journal of Medicine 355(13): 1300-1303.

Kolstad, Jonathan T., and Amanda E. Kowalski. 2010. "The Impact of Health Care Reform On Hospital and Preventive Care: Evidence from Massachusetts." National Bureau of

\footnotetext{
${ }^{10}$ Based on our data, we assume an annual ED visit rate of 0.3 visits per capita for 19-26-year olds. Data on the population of individuals at each age come from the US Census.
} 
Economic Research Working Paper Series No. 16012.

Kriss, Jennifer L et al. 2008. "Rite of passage? Why young adults become uninsured and how new policies can help, 2008 update." Issue Brief (Commonwealth Fund) 38: 1-24.

Levine, Phillip B, Robin McKnight, and Samantha Heep. 2011. "How Effective are Public Policies to Increase Health Insurance Coverage Among Young Adults?” American Economic Journal: Economic Policy 3(1): 129-156.

Marquis, M. Susan, and Stephen H. Long. 1995. "Worker demand for health insurance in the non-group market." Journal of Health Economics 14(1): 47-63.

Miller, Sarah. 2011. "The Effect of Insurance on Emergency Room Visits: An Analysis of the 2006 Massachusetts Health Reform." University of Illinois at Urbana-Champaign Department of Economics Working Paper.

Newton, M. F et al. 2008. "Uninsured Adults Presenting to US Emergency Departments: Assumptions versus Data." Journal of American Medical Association 300(16): 1914.

Trzeciak, S, and E P Rivers. 2003. "Emergency department overcrowding in the United States: an emerging threat to patient safety and public health." Emergency Medicine Journal: EMJ 20(5): 402-405.

White, Patience H. 2002. "Access to health care: health insurance considerations for young adults with special health care needs/disabilities." Pediatrics 110(6 Pt 2): 1328-1335. 
Figure 1. Emergency Department Visits
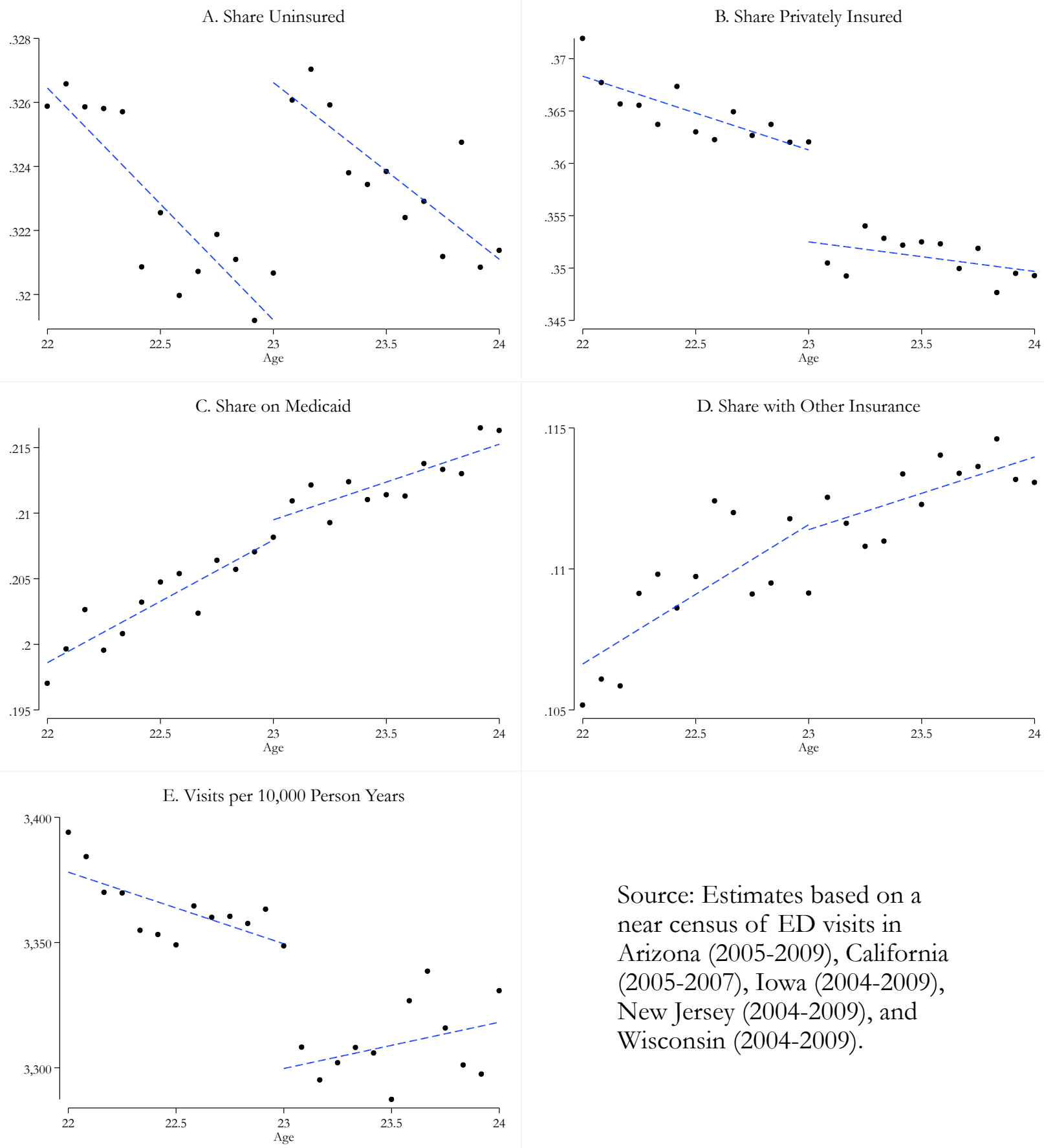

Source: Estimates based on a near census of $\mathrm{ED}$ visits in Arizona (2005-2009), California (2005-2007), Iowa (2004-2009), New Jersey (2004-2009), and Wisconsin (2004-2009). 
Figure 2. Inpatient Visits
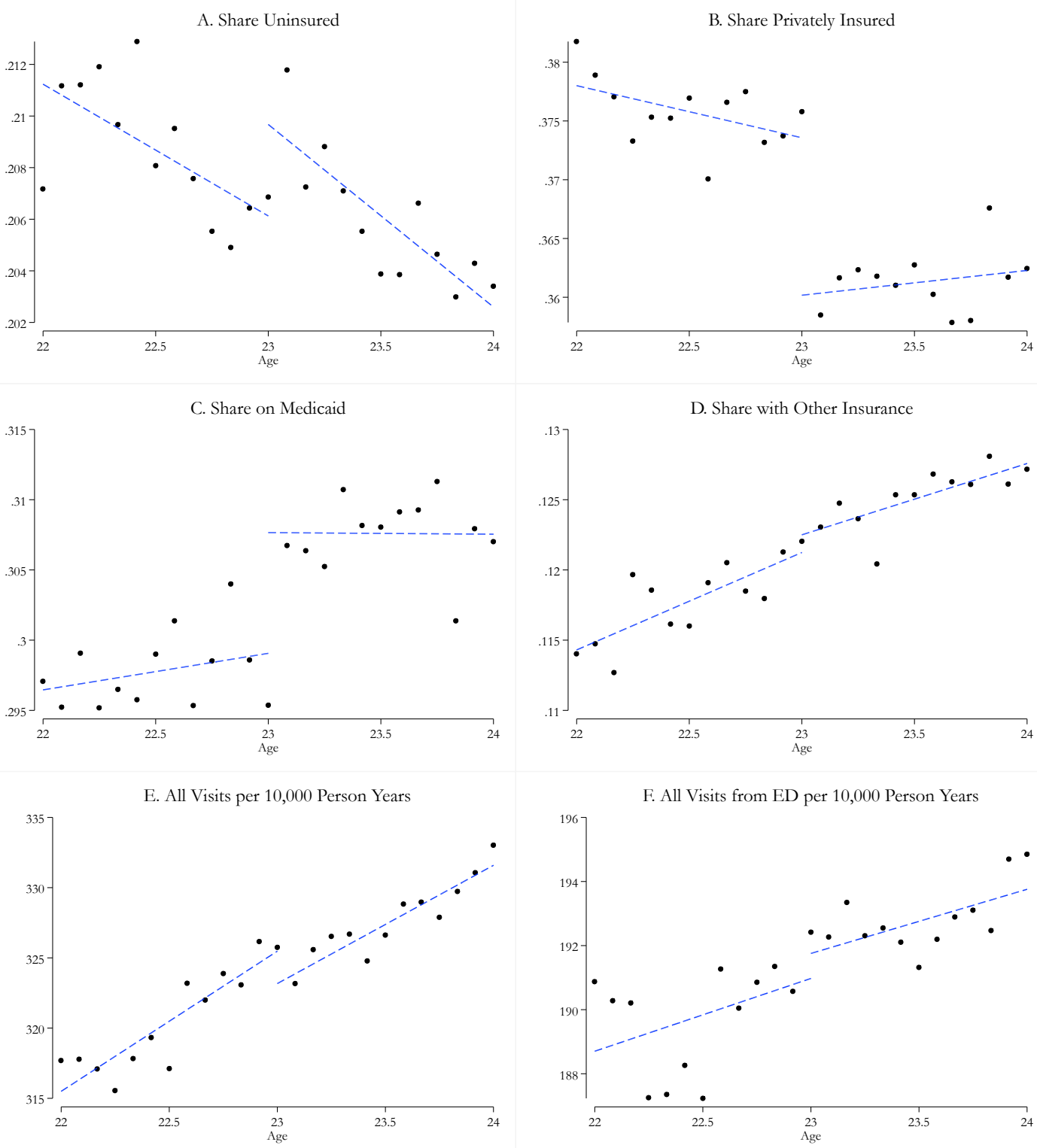

G. All Visits not from ED per 10,000 Person Years

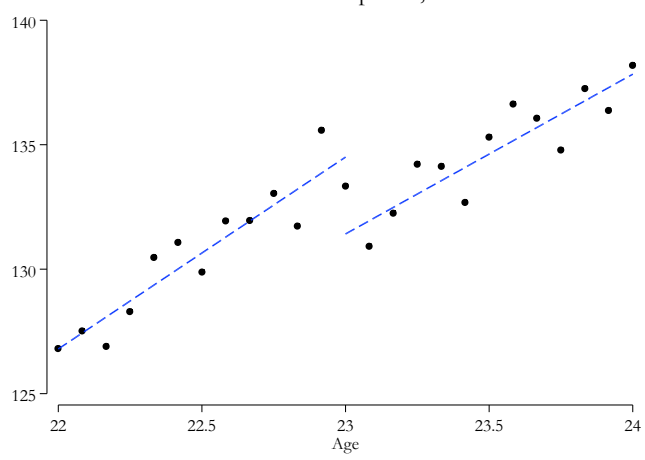

Source: Estimates based on a near census of inpatient visits in Arizona (1990-2009), California (1990-2006), Iowa (2004-2009), New York (1992-2008), Texas (1999-2003) and Wisconsin (2004-2009). The sample is composed of 1,047,605 hospital visits. 
Table 1: Adjusted First Stage at Age 23

\begin{tabular}{lcccc}
\hline & $(1)$ & $(2)$ & $(3)$ & $(4)$ \\
\hline Type of Insurance: & Private & Uninsured & Medicaid & $\begin{array}{c}\text { Other } \\
\text { Insurance }\end{array}$ \\
\hline Sample: & & & & \\
ED Visits & -0.0149 & 0.0181 & -0.0015 & -0.0017 \\
& {$[0.0016]$} & {$[0.0022]$} & {$[0.0012]$} & {$[0.0011]$} \\
& 0.3619 & 0.3191 & 0.2077 & 0.1113 \\
& & & & \\
Inpatient & & & & 0.0003 \\
Stay & -0.0170 & 0.0107 & 0.0060 & {$[0.0013]$} \\
& {$[0.0023]$} & {$[0.0027]$} & {$[0.0022]$} & 0.1212 \\
\hline
\end{tabular}

Notes: Pregnancy related ED visits and hospital stays have been dropped from the analysis. ED visits are a near census of ED visits in Arizona (2005-2009), California (2005-2007), Iowa (2004-2009), New Jersey (20042009) and Wisconsin (2004-2009) and total 2,267,264 visits among 22-23 year olds. Inpatient stays are a near census of hospital admissions in Arizona (1990-2009), California (1990-2006), Iowa (2004-2009), New York (1992-2008), Texas (1999-2003) and Wisconsin (2004-2009) and total 1,047,605 hospital stays. Parameter estimates in the table above represent the percentage point change in insurance coverage when people age out of their insurance coverage on the last day of the month in which they turn 23. Standard errors are in brackets directly below the parameter estimates. The estimated level of the dependent variable, measured immediately before age 23, is below the standard errors. Parameter estimates are adjusted for the decline in admissions under the assumption that the decline in admissions is due entirely to people losing their insurance coverage. The adjustment is made by estimating the insurance coverage regression and the admissions regressions via seemingly unrelated regressions and then using the estimated percent drop in admissions to adjust the coverage estimates. The regressions are run on the averages for one month cells (the most refined version of the age variable available). 
Table 2: Change in ED Visits and Hospital Stays at Age 23

\begin{tabular}{|c|c|c|c|c|}
\hline & $(1)$ & $(2)$ & (3) & (4) \\
\hline & All Visits & $\begin{array}{c}\text { Public } \\
\text { Hospitals } \\
\end{array}$ & $\begin{array}{c}\text { Non Profit } \\
\text { Hospitals }\end{array}$ & $\begin{array}{l}\text { For Profit } \\
\text { Hospitals } \\
\end{array}$ \\
\hline \multicolumn{5}{|l|}{ Outcome: } \\
\hline ED Visits & $\begin{array}{l}-0.0158 \\
{[0.0030]}\end{array}$ & $\begin{array}{l}-0.0091 \\
{[0.0085]}\end{array}$ & $\begin{array}{l}-0.0153 \\
{[0.0036]}\end{array}$ & $\begin{array}{l}-0.0324 \\
{[0.0089]}\end{array}$ \\
\hline All Inpatient & -0.0082 & -0.0035 & -0.0064 & -0.0348 \\
\hline Visits & {$[0.0036]$} & [0.0106] & [0.0046] & [0.0144] \\
\hline $\begin{array}{l}\text { Inpatient Visits } \\
\text { through ED }\end{array}$ & $\begin{array}{c}0.0022 \\
{[0.0054]}\end{array}$ & $\begin{array}{l}-0.0089 \\
{[0.0118]}\end{array}$ & $\begin{array}{c}0.0073 \\
{[0.0074]}\end{array}$ & $\begin{array}{c}0.0065 \\
{[0.0208]}\end{array}$ \\
\hline $\begin{array}{l}\text { Inpatient Visits } \\
\text { Not through ED }\end{array}$ & $\begin{array}{l}-0.0233 \\
{[0.0066]} \\
\end{array}$ & $\begin{array}{r}0.0079 \\
{[0.0191]} \\
\end{array}$ & $\begin{array}{r}-0.0269 \\
{[0.0081]} \\
\end{array}$ & $\begin{array}{l}-0.0703 \\
{[0.0179]} \\
\end{array}$ \\
\hline \multicolumn{5}{|c|}{$\begin{array}{l}\text { Notes: See notes from Table } 2 \text {. The dependent variable in all the regressions above is the log of visits or hospital stays by } \\
\text { month of age. Of the 2,267,264 total emergency department visits among } 22-23 \text { year olds, } 301,664 \text { are to public hospitals, } \\
1,721,554 \text { are to non-profit hospitals, } 213,882 \text { are to for-profit hospitals, and the remaining admissions are to hospitals of } \\
\text { unknown ownership type. Of the 1,045,539 hospital stays that are not pregnancy related, } 617,560 \text { are through the ED and } \\
\text { the remainder are direct admissions to the hospital. Of these 1,045,539 hospital stays, } 239,452 \text { are at public hospitals, } \\
642,991 \text { are at non profit hospitals, } 114,673 \text { are at for profit hospitals and the remainder are at hospitals of unknown type. }\end{array}$} \\
\hline
\end{tabular}


Table 3: Impact of Losing Insurance on Visits at Age 23

\begin{tabular}{lcccc}
\hline & $(1)$ & $(2)$ & $(3)$ & $(4)$ \\
\hline & & Public & Non Profit & For Profit \\
& All Visits & Hospitals & Hospitals & Hositals \\
\hline Outcome: & & & & -1.764 \\
ED Visits & -0.867 & -0.503 & -0.838 & {$[0.539]$} \\
\hline
\end{tabular}

Notes: See notes from Tables 1 and 2. Each coefficient is the ratio of a "reduced form" coefficient from Table 2 divided by its associated "first stage" coefficient from Table 1. Standard errors are computed using the Delta Method. 